An International Journal of Optimization and Control: Theories \& Applications ISSN:2146-0957 eISSN:2146-5703

Vol.7, No.3, pp.255-259 (2017)

http://doi.org/10.11121/ijocta.01.2017.00498

RESEARCH ARTICLE

\title{
Symmetry solution on fractional equation
}

\author{
Gulistan Iskandarova*, Dogan Kaya \\ Department of Mathematics, Istanbul Commerce University, Istanbul, Turkey \\ gulistan.iskandarova@gmail.com,dogank@ticaret.edu.tr
}

\begin{tabular}{|c|c|}
\hline ARTICLE INFO & ABSTRACT \\
\hline $\begin{array}{l}\text { Article History: } \\
\text { Received } 29 \text { June } 2017 \\
\text { Accepted } 23 \text { October } 2017 \\
\text { Available } 25 \text { October } 2017 \\
\end{array}$ & $\begin{array}{l}\text { As we know nearly all physical, chemical, and biological processes in nature } \\
\text { can be described or modeled by dint of a differential equation or a system of } \\
\text { differential equations, an integral equation or an integro-differential equation. } \\
\text { The differential equations can be ordinary or partial, linear or nonlinear. So, }\end{array}$ \\
\hline $\begin{array}{l}\text { Keywords: } \\
\text { Riemann-Liouville fractional derivative } \\
\text { Lie groups } \\
\text { Mittag-Leffler function }\end{array}$ & $\begin{array}{l}\text { we concentrate our attention in problem that can be presented in terms of a } \\
\text { differential equation with fractional derivative. Our research in this work is to } \\
\text { use symmetry transformation method and its analysis to search exact solutions } \\
\text { to nonlinear fractional partial differential equations. }\end{array}$ \\
\hline
\end{tabular}

AMS Classification 2010:

26A33, 54H15, 33E12

(cc) $\mathrm{BY}$

\section{Introduction}

The fractional derivatives that are about three centuries ago were presented [1,2. Fractional differential equations have been used successfully to describe many complex nonlinear phenomena and dynamic processes in physics, electromagnetics [3, 4], acoustics, astrophysics [5, 6], viscoelasticity, chemistry, electrochemistry, etc. [1, 2, 7, 8]. As for the methods for solving such equations, there is no effective general method. But there have been formulated and applied methods like Adomian decomposition method [9], differential transform method [10, modified simple equation method [11,12], Lie symmetry analysis [13,14] and so on.

Lie symmetry analysis is powerful and universal tool for searching solution of linear and nonlinear partial differential equations and it has been widely applied for studying the invariance properties of partial differential equation (PDE) 15 . A symmetry of a PDE is any transformation that each solution surface of the PDE is mapped to another solution surface of the same PDE, i.e. leaves invariant its solution space. So, by using the Lie symmetry, the equation can be transformed into a nonlinear fractional ODE.

For construction a symmetry reductions of a fractional equation we investigated the symmetry properties by using the group analysis method and presented the vector fields the equation based on the point symmetry [13, 14]. It is shown that our equation could be transformed into a nonlinear fractional ordinary differential equation with the new independent variable.

In this work by using the Lie group, we investigate the symmetry properties of fractional partial differential equation (FPDE)

$$
\frac{\partial^{\alpha} u}{\partial t^{\alpha}}=\left(g(u) u_{x x}\right)_{x}
$$

and find the correspondence infinitesimal operators and then construct some exact solution of these equations, in particulary the solution for fractional linear KdV equation.

The outline of this paper is as follows: in section 2 we will give general definitions and formulas of fractional derivative and symmetry analysis, also we show the application of symmetry group to

*Corresponding Author 
fractional differential Eq. (11). In section 3 presented some exact solutions by using symmetry reductions.

\section{Preliminaries}

\subsection{Lie symmetry analysis of fractional PDE}

Consider a time FPDE with two independent variables and $0<\alpha \leq 1$ is given as following:

$$
\frac{\partial^{\alpha} u}{\partial t^{\alpha}}-\left(g(u) u_{x x}\right)_{x}=0
$$

here fractional derivative are considered in the Riemann-Liouville terms.

Suppose $f$ be integrable on $[a, b] \in \mathbb{R}$ and $n-1<$ $\alpha<n, n \in \mathbb{N}$. Then Riemann-Liouville fractional derivative is defined as

$$
\begin{aligned}
{ }_{0} D_{t}^{\alpha} f(t) & =\frac{\partial^{\alpha} f(t)}{\partial t^{\alpha}} \\
& =\frac{1}{\Gamma(n-\alpha)} \frac{\partial^{n}}{\partial t^{n}} \int_{0}^{t}(t-\tau)^{n-\alpha-1} f(\tau) d \tau .
\end{aligned}
$$

Let $f$ be integrable on $[0, \infty)$, and piecewise continuous function on $(0, \infty)$ and $\operatorname{Re} \alpha>0, t>0$. Then Riemann-Liouville fractional integral is defined by

$$
{ }_{0} I_{t}^{\alpha} f(t)=\frac{1}{\Gamma(\alpha)} \int_{0}^{t}(t-x)^{\alpha-1} f(x) d x .
$$

One parameter Lie symmetry transformations are determined as

$$
\begin{aligned}
& \bar{t}=t+\varepsilon \tau(x, t, u)+O\left(\varepsilon^{2}\right), \\
& \bar{x}=x+\varepsilon \xi(x, t, u)+O\left(\varepsilon^{2}\right), \\
& \bar{u}=u+\varepsilon \eta(x, t, u)+O\left(\varepsilon^{2}\right),
\end{aligned}
$$

where $\epsilon>0$ is a infinitesimals parameter with $\xi=\left.\frac{d \bar{x}}{d \epsilon}\right|_{\epsilon=0}, \tau=\left.\frac{d \bar{t}}{d \epsilon}\right|_{\epsilon=0}$ and $\eta=\left.\frac{d \bar{u}}{d \epsilon}\right|_{\epsilon=0}$ which will be determined.

After applying transformation (5) to usual partial derivatives $u_{x}, u_{x x}$ and $u_{x x x}$ it gives the following extensions [15]:

$$
\begin{aligned}
& \frac{\partial \bar{u}}{\partial x}=\frac{\partial u}{\partial x}+\varepsilon \eta_{1}^{x}+O\left(\varepsilon^{2}\right), \\
& \frac{\partial^{2} \bar{u}}{\partial x^{2}}=\frac{\partial^{2} u}{\partial x^{2}}+\varepsilon \eta_{2}^{x}+O\left(\varepsilon^{2}\right), \\
& \frac{\partial^{3} \bar{u}}{\partial x^{3}}=\frac{\partial^{3} u}{\partial x^{3}}+\varepsilon \eta_{3}^{x}+O\left(\varepsilon^{2}\right) .
\end{aligned}
$$

Here $\eta_{1}^{x}, \eta_{2}^{x}$ and $\eta_{3}^{x}$ are defined by formulae

$$
\begin{aligned}
& \eta_{1}^{x}=D_{x} \eta-u_{x} D_{x} \xi-u_{t} D_{x} \tau, \\
& \eta_{2}^{x}=D_{x} \eta_{1}^{x}-u_{x x} D_{x} \xi-u_{x t} D_{x} \tau, \\
& \eta_{3}^{x}=D_{x} \eta_{2}^{x}-u_{x x x} D_{x} \xi-u_{x x t} D_{x} \tau,
\end{aligned}
$$

where $D_{x}$ is the total derivative

$$
D_{x}=\frac{\partial}{\partial x}+u_{x} \frac{\partial}{\partial u}+u_{x t} \frac{\partial}{\partial u_{t}}+u_{x x} \frac{\partial}{\partial u_{x}}+\cdots .
$$

And the $\alpha$ th extended infinitesimal related to Riemann-Liouville fractional time derivative is [16] as

$$
\frac{\partial^{\alpha} \bar{u}}{\partial t^{\alpha}}=\frac{\partial^{\alpha} u}{\partial t^{\alpha}}+\varepsilon \eta_{\alpha}^{t}+O\left(\varepsilon^{2}\right) .
$$

Here $\eta_{\alpha}^{t}$ has following form:

$$
\begin{aligned}
\eta_{\alpha}^{t}=D_{t}^{\alpha}(\eta) & +\xi D_{t}^{\alpha}\left(u_{x}\right)-D_{t}^{\alpha}\left(\xi u_{x}\right)+D_{t}^{\alpha}\left(u D_{t} \tau\right) \\
& -D_{t}^{\alpha+1}(\tau u)+\tau D_{t}^{\alpha+1} u
\end{aligned}
$$

and the operator $D_{t}^{\alpha}$ is the total fractional derivative operator. Using the generalized Leibnitz rule 2

$$
\begin{aligned}
& D_{t}^{\alpha}(f(t) g(t))=\sum_{n=0}^{\infty}\left(\begin{array}{l}
\alpha \\
n
\end{array}\right) D_{t}^{\alpha-n} f(t) D_{t}^{n} g(t), \\
& \left(\begin{array}{l}
\alpha \\
n
\end{array}\right)=\frac{(-1)^{n-1} \alpha \Gamma(n-\alpha)}{\Gamma(1-\alpha) \Gamma(n+1)} .
\end{aligned}
$$

Thus infinitesimal $\eta_{\alpha}^{t}$ is modified to

$$
\begin{aligned}
\eta_{\alpha}^{t} & =\frac{\partial^{\alpha} \eta}{\partial t^{\alpha}}+\left(\eta_{u}-\alpha\left(\tau_{t}+u_{t} \tau_{u}\right)\right) \frac{\partial^{\alpha} u}{\partial t^{\alpha}}-u \frac{\partial^{\alpha} \eta_{u}}{\partial t^{\alpha}}+\mu \\
& +\sum_{n=1}^{\infty}\left[\left(\begin{array}{l}
\alpha \\
n
\end{array}\right) \frac{\partial^{n} \eta_{u}}{\partial t^{n}}-\left(\begin{array}{c}
\alpha \\
n+1
\end{array}\right) D_{t}^{\alpha+1} \tau\right] D^{\alpha-n} u \\
& -\sum_{n=1}^{\infty}\left(\begin{array}{l}
\alpha \\
n
\end{array}\right)\left(D_{t}^{n} \xi\right)\left(D_{t}^{\alpha-n} u_{x}\right) .
\end{aligned}
$$

The corresponding vector field $V$ associated with transformations (5) can be written as

$$
V=\xi(x, t, u) \partial_{x}+\tau(x, t, u) \partial_{t}+\eta(x, t, u) \partial_{u} .
$$

Applying the third prolongation $p r^{(3)} V$ to Eq. (2), we will get

$$
\left.p r^{(3)} V(\Delta)\right|_{\Delta=0}=0, \quad \Delta=\frac{\partial^{\alpha} u}{\partial t^{\alpha}}-\left(k(u) u_{x x}\right)_{x},
$$


where the operator $p^{(3)} V$ takes the following form:

$p^{(3)} V=V+\eta_{\alpha}^{t} \partial_{\partial_{t}^{\alpha} u}+\eta_{1}^{x} \partial_{u_{x}}+\eta_{2}^{x} \partial_{u_{x x}}+\eta_{3}^{x} \partial_{u_{x x x}}$.

Our equation (2) can be written in the form

$$
\frac{\partial^{\alpha} u}{\partial t^{\alpha}}-g^{\prime}(u) u_{x x} u_{x}-g(u) u_{x x x}=0 .
$$

Substitution of transformations (5), (6) and (8) into (11) we get

$$
\begin{aligned}
& \frac{\partial^{\alpha} \bar{u}}{\partial \bar{t}^{\alpha}}-g^{\prime}(\bar{u}) \bar{u}_{\bar{x} \bar{x}} \bar{u}_{\bar{x}}-g(\bar{u}) \bar{u}_{\bar{x} \bar{x} \bar{x}} \\
& =\frac{\partial^{\alpha} u}{\partial t^{\alpha}}-g^{\prime}(u) u_{x x} u_{x}-g(u) u_{x x x} \\
& +\epsilon\left(\eta_{\alpha}^{t}-\eta\left(g^{\prime \prime} u_{x} u_{x x}+g^{\prime} u_{x x x}\right)\right. \\
& \left.-g^{\prime} u_{x} \eta_{2}^{x}-g^{\prime} u_{x x} \eta_{1}^{x}-g \eta_{3}^{x}\right)+O\left(\epsilon^{3}\right)
\end{aligned}
$$

So we find that the functions $\xi(x, t, u), \tau(x, t, u)$ and $\eta(x, t, u)$ must satisfy the symmetry condition

$$
\eta_{\alpha}^{t}-\eta\left(g^{\prime \prime} u_{x} u_{x x}+g^{\prime} u_{x x x}\right)-g^{\prime} u_{x} \eta_{2}^{x}-g^{\prime} u_{x x} \eta_{1}^{x}-g \eta_{3}^{x}=0 .
$$

Solving the Eq. (12) along with Eq. (21) and substituting the extended infinitesimal (77), (9) into the Eq. (12) we get following characteristic system:

$$
\begin{aligned}
& \xi_{u}=\xi_{t}=\tau_{u}=\tau_{x}=\eta_{u u}=0, \\
& -\alpha g^{\prime} \tau_{t}-g^{\prime \prime} \eta+3 g^{\prime} \xi_{x}-g^{\prime} \eta_{u}=0, \\
& -\alpha g \tau_{t}-g^{\prime} \eta+3 g \xi_{x}=0, \\
& -g^{\prime} \eta_{x x}-3 g \eta_{x x u}=0, \\
& -2 g^{\prime} \eta_{x u}+g^{\prime} \xi_{x x}=0, \\
& -g^{\prime} \eta_{x}-3 g \eta_{x u}+3 g \xi_{x x}=0 \\
& \eta_{u t}-\frac{\alpha-1}{2} \tau_{t t}=0 \\
& \frac{\partial^{\alpha} \eta}{\partial t^{\alpha}}-u \frac{\partial^{\alpha} \eta_{u}}{\partial t^{\alpha}}-g \eta_{x x x}=0 .
\end{aligned}
$$

Solving these equations we investigate generating infinitesimal operators as following.

Case 1: For arbitrary $g(u)$ and $0<\alpha \leq 1$ there are three infinitesimal operators

$$
X_{1}=\frac{\partial}{\partial x}, \quad X_{2}=\frac{\partial}{\partial t}, \quad X_{3}=x \frac{\partial}{\partial x}+\frac{3 t}{\alpha} \frac{\partial}{\partial t} .
$$

Case 2: For $g(u)=1$ and $0<\alpha \leq 1$ there are two additional infinitesimal operators

$$
X_{4}=u \frac{\partial}{\partial u}, \quad X_{\infty}=h(t, x) \frac{\partial}{\partial u},
$$

where the function $h(t, x)$ satisfies the linear fractional KdV equation $D_{t}^{\alpha} h=h_{x x x}$.

Case 3: For $g(u)=u^{b}$ with $b \neq 0$ and $0<\alpha \leq 1$ there are two additional infinitesimal operators

$$
X_{4}=x \frac{\partial}{\partial x}+\frac{3 u}{b} \frac{\partial}{\partial u}, \quad X_{5}=t \frac{\partial}{\partial t}-\frac{\alpha u}{b} \frac{\partial}{\partial u} .
$$

Case 4: For $g(u)=u^{b}$ with $b=-3$ and $0<\alpha \leq 1$ there is one additional infinitesimal operator

$$
X_{6}=x \frac{\partial}{\partial x}-\frac{6 t}{\alpha-3} \frac{\partial}{\partial t}-\frac{(3 \alpha-3) u}{\alpha-3} \frac{\partial}{\partial u} .
$$

Case 5: For $g(u)=e^{u}$ with integer $\alpha(\alpha=1)$ there are two additional infinitesimal operators

$$
X_{4}=x \frac{\partial}{\partial x}+3 \frac{\partial}{\partial u}, \quad X_{5}=t \frac{\partial}{\partial t}-\alpha \frac{\partial}{\partial u} .
$$

Theorem 1. The equation $D_{t}^{\alpha} u=\left(g(u) u_{x x}\right)_{x}$ with $g(u)=e^{u}$ and $0<\alpha<1$ has no additional symmetries.

Proof. For $g(u)=e^{u}$ and $0<\alpha<1$ the system (13) transforms to

(1) $\xi_{u}=\xi_{t}=\tau_{u}=\tau_{x}=\eta_{u u}=0$,

(2) $-\alpha e^{u} \tau_{t}-e^{u} \eta+3 e^{u} \xi_{x}-e^{u} \eta_{u}=$ 0

(3) $-\alpha e^{u} \tau_{t}-e^{u} \eta+3 e^{u} \xi_{x}=0$,

(4) $-e^{u} \eta_{x x}-3 e^{u} \eta_{x x u}=0$,

(5) $-2 e^{u} \eta_{x u}+e^{u} \xi_{x x}=0$,

(6) $-e^{u} \eta_{x}-3 e^{u} \eta_{x u}+3 e^{u} \xi_{x x}=0$,

(7) $\eta_{u t}-\frac{\alpha-1}{2} \tau_{t t}=0$,

(8) $\frac{\partial^{\alpha} \eta}{\partial t^{\alpha}}-u \frac{\partial^{\alpha} \eta_{u}}{\partial t^{\alpha}}-e^{u} \eta_{x x x}=0$.

The first equation gives us that $\xi=A(x), \tau=$ $B(t)$ and $\eta=C(x, t) u+D(x, t)$. So from 2. and 3 . equations we get $C=0$, also from 5 . and 7 . equations we find $A=c_{1} x+c_{2}$ and $B=c_{3} t+c_{4}$. Thus by finding the corresponding derivatives and putting them to equation 2 . we find that $D$ is constant, but from 8. equation $\frac{\partial^{\alpha} D}{\partial t^{\alpha}}=0$, which gives us $D=c_{5} t^{\alpha-1}$ thereby we have obtained a contradiction. It means that for $0<\alpha<1$ and $g(u)=e^{u}$ there is not any additional symmetries. 


\section{Symmetry reductions and some exact solutions}

\subsection{The exact solution for $g(u)=1$}

For $g(u)=1$ we have linear fractional KdV equation $D_{t}^{\alpha} u=u_{x x x}$ with infinitesimal operators

$$
\begin{gathered}
X_{1}=\frac{\partial}{\partial x}, \quad X_{2}=\frac{\partial}{\partial t}, \quad X_{3}=x \frac{\partial}{\partial x}+\frac{3 t}{\alpha} \frac{\partial}{\partial t}, \\
X_{4}=u \frac{\partial}{\partial u}, \quad X_{\infty}=h(t, x) \frac{\partial}{\partial u} .
\end{gathered}
$$

By composition of $X_{1}$ and $X_{4}$ we get generator

$$
X_{1}+k X_{4}=\frac{\partial}{\partial x}+k u \frac{\partial}{\partial u},
$$

where $k \in \mathbb{R}$. Then solution under the group has the form $u(t, x)=e^{k x} \phi(t)$, where $\phi(t)$ satisfies the equation

$$
D_{t}^{\alpha} \phi(t)=k^{3} \phi(t)
$$

and thus

$$
u(t, x)=e^{k x} t^{\alpha-1} E_{\alpha, \alpha}\left(k^{3} t^{\alpha}\right) .
$$

Here $E_{\alpha, \beta}(x)$ is a Mittag-Leffler function

$$
E_{\alpha, \beta}(x)=\sum_{m=0}^{\infty} \frac{x^{m}}{\Gamma(\alpha m+\beta)} .
$$

\subsection{The exact solution for $g(u)=u^{b}$}

For $g(u)=u^{b}$ we have $D_{t}^{\alpha} u=b u^{b-1} u_{x x} u_{x}+$ $u^{b} u_{x x x}$ with infinitesimal operators

$$
\begin{aligned}
& X_{1}=\frac{\partial}{\partial x}, \quad X_{2}=\frac{\partial}{\partial t}, \quad X_{3}=x \frac{\partial}{\partial x}+\frac{3 t}{\alpha} \frac{\partial}{\partial t} \\
& X_{4}=x \frac{\partial}{\partial x}+\frac{3 u}{b} \frac{\partial}{\partial u}, \quad X_{5}=t \frac{\partial}{\partial t}-\frac{\alpha u}{b} \frac{\partial}{\partial u} .
\end{aligned}
$$

With $X_{4}=x \frac{\partial}{\partial x}+\frac{3 u}{b} \frac{\partial}{\partial u}$ solution under the group has the form

$$
u(t, x)=x^{3 / b} \phi(t),
$$

where $\phi(t)$ satisfies the equation

$$
D_{t}^{\alpha} \phi(t)=\frac{3\left(9-b^{2}\right)}{b^{3}} \phi^{b+1}(t) .
$$

If $b=3$ then we derive $D_{t}^{\alpha} \phi(t)=0$, which gives $\phi(t)=C t^{\alpha-1}, \quad C \in \mathbb{R}$. Therefore

$$
u(x, t)=C x^{3 / b} t^{\alpha-1} .
$$

Also if $b=-1$ then $D_{t}^{\alpha} \phi(t)=-24$, which gives

$$
u(t, x)=-\frac{24 x^{-3} t^{\alpha}}{\Gamma(\alpha+1)} \text {. }
$$

\section{Conclusion}

For construction a symmetry reductions of the fractional equation (1) we investigated the symmetry properties by using the symmetry analysis method and presented different infinitesimal operators. We obtained solutions for two particular equations with some generator operators. Also we showed that the equation $D_{t}^{\alpha} u=\left(e^{u} u_{x x}\right)_{x}$ for $0<\alpha<1$ has only general symmetries with $X_{1}=\frac{\partial}{\partial x}, \quad X_{2}=\frac{\partial}{\partial t}, X_{3}=x \frac{\partial}{\partial x}+\frac{3 t}{\alpha} \frac{\partial}{\partial t}$ infinitesimal operators. The symmetry analysis or Lie group analysis is a very powerful method and is worthy of studying further to searching the solutions and symmetry properties of nonlinear partial differential equations and fractional nonlinear partial differential equations.

\section{References}

[1] Diethelm, K. (2010). The analysis of fractional differential equations. Springer, Berlin.

[2] Miller, K.S., Ross, B. (1993). An introduction to the fractional calculus and fractional differential equations. Wiley, New York.

[3] Zelenyi, L.M., Milovanov, A.V. (2004). Fractal topology and strange kinetics: from percolation theory to problems in cosmic electrodynamics. Physics Uspekhi, 47, 749-788.

[4] Bulut, H., Yel, G., Baskonus, H.M. (2016). An application of improved Bernoulli sub-equation function method to the nonlinear time-fractional Burgers equation. Turkish Journal of Mathematics and Computer Science, 5, 1-17.

[5] Tarasov, V.E. (2006). Gravitational field of fractal distribution of particles. Celes. Mech. Dynam. Astron., $19,1-15$.

[6] Kaya, D., Bulut, H. (2000). The decomposition method for approximate solution of a burgers equation. Bulletin of the Institute of Mathematics Academia Sinica, 28, 35-42.

[7] Wu, G., Lee, E.W.M. (2010) Fractional variational iteration method and its application. Phys. Lett. A., 374, 2506-2509.

[8] Kaya, D., Bulut, H. (2000). On a comparison between decomposition method and one-step methods for nonlinear initial-value problems. Journal of natural and engineering sciences, 12(2), 299-305.

[9] Barley, R., Torvik, P. (1983). A theoretical basis for the application of fractional calculus to viscoelasticity. J. Rheol., 27, 201-210. 
[10] Hereman, W., Banerjee, P.P., Chatterjee, M.R. (1989). On the nonlocal equations and nonlocal charges associated with the Harry-Dym hierarchy Korteweg-de-Vries equation. J. Phys. A., 22, 241-252.

[11] Taghizadeh, N., Mirzazadeh, M., Rahimian, M., Akbari, M. (2013). Application of the simplest equation method to some time-fractional partial differential equations. Ain Shams Eng. J., 4(4), 897-902.

[12] Baskonus, H.M., Mekkaoui, T., Hammouch, Z., Bulut, H. (2015) Active control of a chaotic fractional order economic system. Entropy, 17(8), 5771-5783.

[13] Hu, J., Ye, Y., Shen, S., Zhang, J. (2014). Lie symmetry analysis of the time fractional $\mathrm{dV}$-type equation. Appl. Math. and Comp., 223, 439-444.

[14] Sahadevan, R., Bakkyaraj, T. (2012). Invariant analysis of time fractional generalized Burgers and Kortewegde Vries equations. J. Math. Anal. Appl., 393, 341-347.

[15] Bluman, G.W., Anco, S. (2002). Symmetry and integration methods for differential equations. SpringerVerlag, Heidelburg.

[16] Gazizov, R.K., Kasatkin, A.A., Lukashchuk, S.Y. (2007). Continuous transformation group of fractional differential equations. Vestn. USATU, 9, 125-135.
Gulistan Iskandarova is a Ph.D. Student at Istanbul Commerce University. She received her M.Sc. in Eurasian National University in Kazakhstan, Astana. Her research interests are nonlinear ordinary differential equations partial differential equations, fractional derivative theory, analytical methods for the nonlinear differential equations, Painleve transients, Lie groups theory. She is author and co-author of several papers, some of which appeared in Journal Republican Student Scientific Conference on Mathematics, Mechanics and Computer Science and Journal International Scientific Conference of students, graduate students and young scientists "Lomonosov".

Dogan Kaya is currently professor in Department of Mathematics and Computer Science, Istanbul Commerce University. He received his PhD degree from University of Newcastle-upon-Tyne (England) in 1995. His research area includes numerical analysis, nonlinear ordinary differential equations partial differential equations, analytical methods for nonlinear differential equations and numerical solutions of the partial differential equations, mathematical programming.

An International Journal of Optimization and Control: Theories \& Applications (http://ijocta.balikesir.edu.tr)

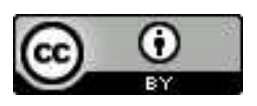

This work is licensed under a Creative Commons Attribution 4.0 International License. The authors retain ownership of the copyright for their article, but they allow anyone to download, reuse, reprint, modify, distribute, and/or copy articles in IJOCTA, so long as the original authors and source are credited. To see the complete license contents, please visit http://creativecommons.org/licenses/by/4.0/. 